\title{
Analysis of the influence of positive feedback on the quality of the control system
}

\author{
Irina Tsybriy ${ }^{1, *}$, Vitaliy Ignatenko ${ }^{2}$ \\ ${ }^{1}$ Department of Instrument-making, Don State Technical University, Russia \\ ${ }^{2}$ Department of Instrument-making, Don State Technical University, Russia
}

\begin{abstract}
The paper proposed a matrix mathematical apparatus of synthesis optimal drive control system, providing the change of the angular acceleration of the output shaft for a given law. An example is given of constructing a matrix model for optimal control of a high-torque direct current drive with thyristor control.
\end{abstract}

\section{Introduction}

The conditions for high-performance equipment require the use of drives built on the principle of optimal control [1 - 7] by the criterion of maximum speed. The control criterion can be formulated as follows $[8,9]$ "... the optimum speed is achieved in those cases when the selected executive motor for a given typical control action develops the maximum possible torque in the required direction during the entire time of the transient process."

As you know [3], the drive with the maximum speed has a step-like control action, performed in two stages:

- the first one accelerates the engine with the maximum possible acceleration and the engine speed changes by a value $\Delta \Omega$;

- on the second - braking of the engine with the maximum possible deceleration and speed change by the value $\Delta \Omega$.

Depending on the parameters of the drive, the value of the control time $t$ and the set value of the movement $\mathrm{S}$, the following modes of playback of this path are possible:

- when acceleration is limited; acceleration is limited by the maximum torque of the motor, the static torque of the load and the moment of inertia of the drive;

- under the restriction of acceleration and the derivative of acceleration; the derivative of acceleration is limited by the maximum rate of change of the torque in the function of time;

- when speed and acceleration are limited; speed is limited by the maximum engine speed for a given load value;

- with the limitation of speed, acceleration and derivative of acceleration.

The limitation of the rate of change of the motor torque in time is of considerable importance only at a low moment of inertia of the drive. Let us consider the possibility of reproducing a given trajectory of motion with an acceleration limitation.
To analyze the behavior of a system with viscous friction and a nonlinear dependence of the moment of resistance on the angle of rotation of the motor shaft, we consider an electric direct current drive with a hightorque electric motor with a control system built on the principle of subordinate regulation.

\section{Statement of the problem of optimal control}

For example, such a system can always be represented by a system of three linear differential equations of the third order, starting from the form of the transfer function [10]:

$W(p)=\frac{1}{K_{C}} \quad \frac{1+8 \tau p}{64 p^{3} \tau^{3}+32 p^{2} \tau^{2}+8 p \tau+1}=\frac{\omega_{1}(p)}{\theta_{U}(p)}$

where: $\tau$ - time constant of the pulse-phase control system of the thyristor rectifier; $K_{C}$ - coefficient of speed feedback; $\quad \omega_{1}$ - frequency of rotation of the motor shaft; $\theta_{U}-$ voltage at the motor armature (control signal).

The control problem is formulated as follows: drive control must ensure that the angular acceleration of the output shaft is changed according to a given law $\varepsilon=\varepsilon(t)$. The mechanical part of the drive can be represented by a two-mass model.

We denote the law of change of the control action as $\theta(t)$.

The control criterion can be written in the form:

$$
J_{U}=\int_{0}^{\tau}\left[\theta(t)-\varphi_{2}\right]^{2} d t \Rightarrow \min
$$

where: $\varphi_{2}$ - angle of rotation of the actuator, reduced to the shaft of the electric motor.

Obviously, the input action can be specified either by the angle of rotation of the motor shaft as a function of time, or by the angular acceleration as a function of time.

\footnotetext{
* Corresponding author: irconst@mail.ru
} 
The control criterion in matrix form [11 - 15]:

$$
J=\int_{0}^{\infty}\left\{\mathbf{X}^{T} \mathbf{Q X}+\mathbf{U}^{T} \mathbf{R} \mathbf{U}-2 \mathbf{X}^{T} \mathbf{P} \mathbf{U}\right\} d t \Rightarrow \min
$$

where: $\mathbf{X}$ - n-dimensional state vector of the control object; $\mathbf{U}$ - n-dimensional vector of the state of control actions.

The choice of matrices $\mathbf{P}$ and $\mathbf{Q}$ is determined by the type of the optimization criterion, but the transition to the matrix form of recording with the previous formulation of the problem is impossible, so we will carry out its correction.

We consider that the required law of change of control action is the law of change of voltage on the armature of the motor. In this formulation, the system can be described in state variables.

In our example, the equations of a system consisting of a controlled thyristor rectifier, a DC motor, and a mechanical part replaced by an equivalent two-mass model have the following form:

$$
\begin{aligned}
& \dot{\omega}_{1}=y \\
& \dot{y}=z \\
& \dot{z}=\theta(t) a+b z+c y+d \omega_{1} \\
& \dot{M}_{12}=q\left(\omega_{1}-\omega_{2}\right) \\
& \dot{\omega}_{2}=\left(M_{12}-M_{C}\left(\varphi_{2}\right)-\beta \omega_{2}\right) s \\
& \dot{\varphi}_{2}=\omega_{2}
\end{aligned}
$$

where: $a=\frac{K_{C}}{64 \tau^{3}} ; q=\frac{1}{J_{1}} ; b=\frac{1}{2 \tau} ; s=\frac{1}{J_{2}}$;

$c=\frac{1}{8 \tau^{2}} ; \quad r=8 \tau ; d=\frac{1}{64 \tau^{3}} ; \beta$ - coefficient of viscous friction in the actuator; $M c$ - the moment of resistance, $M_{12}\left(\varphi_{2}\right)$ - dependence of the moment of resistance on the angle of rotation of the actuator, reduced to the shaft of the electric motor, $J_{l}, J_{2}$ moments of inertia reduced to the motor shaft.

Then the vector of control actions will take equal:

$$
\mathbf{U}(t)=\left(\begin{array}{c}
\varepsilon(t) \\
-M_{C}\left(\varphi_{2}\right)
\end{array}\right)
$$

\section{Solution of the problem by dynamic programming method}

To solve the problem, we use the method of dynamic programming, which is widely used to solve problems of optimal control [16 - 21]. It is based on the principle: any terminal part of the optimal trajectory is the optimal trajectory. This means that the optimal control is determined by the state of the system at a given moment in time and does not depend on the states at the previous moments of time.

Consider this method in application to an object described in the space of state variables. In our case, the initial state of the object $\mathbf{X}(0)$, the domain of admissible controls $\Psi(\mathbf{U})$ and the optimality criterion are given.

We assume that the upper limit of integration $\tau$ is given. We do not impose constraints on the state vector and its final value of constraints. The argument in the formulas $F(\mathbf{X}, \mathbf{U}, t)$ and $\mathbf{U}(t)$, means that they can be non-stationary.

In this formulation, we can formulate the following problem: it is necessary to find in the class of admissible controls a control $\mathbf{U}(t)$ under which the functional reaches a minimum.

The task is related to the type of tasks with a free right end and a fixed control time: $J=\min$, when: $\mathbf{U}(t) \in \Psi(t) ; t \in\left[t_{0}, T\right]$.

We write the Bellman equation:

$$
-\frac{\partial S}{\partial t}=\min \left\{F(\mathbf{X}, \mathbf{U}, t)+\sum_{k=1}^{n} \frac{\partial S}{\partial x_{k}} \cdot f_{k}\right\}
$$

and perform the following transformations:

$$
\begin{aligned}
& -\frac{\partial S}{\partial t}=\min \left\{\mathbf{X}^{\mathbf{T}} \mathbf{Q X}+\mathbf{U}^{\mathbf{T}} \mathbf{R} \mathbf{U}-2 \mathbf{X}^{\mathbf{T}} \cdot \mathbf{U}+\frac{\partial S}{\partial \mathbf{X}} \cdot(\mathbf{A X}+\mathbf{B U})\right\}- \\
& -\frac{\partial}{\partial \mathbf{U}}\left\{\mathbf{X}^{\mathbf{T}} \mathbf{Q X}+\mathbf{U}^{\mathbf{T}} \mathbf{R} \mathbf{U}-2 \mathbf{X}^{\mathbf{T}} \mathbf{P} \mathbf{U}+\frac{\partial S}{\partial \mathbf{X}} \cdot(\mathbf{A X}+\mathbf{B U})\right\}=0
\end{aligned}
$$

where A - own matrix of the object.

Further we receive:

$$
2 \mathbf{U}^{\mathbf{T}} \cdot \mathbf{R}-2 \cdot \mathbf{X}^{\mathbf{T}} \cdot \mathbf{P}+\frac{\partial S}{\partial \mathbf{X}} \cdot \mathbf{B}=0 .
$$

From the last equality we find the expression for the control vector. For further reasoning we need expressions for $\mathbf{U}$ and $\mathbf{U}^{\mathbf{T}}$ :

$$
\begin{gathered}
\mathbf{U}^{\mathbf{T}}=\left(\mathbf{X}^{\mathbf{T}} \cdot \mathbf{P}-\frac{1}{2} \cdot \frac{\partial S}{\partial \mathbf{X}} \cdot \mathbf{B}\right) \cdot \mathbf{R}^{-1} \\
\mathbf{R}^{\mathbf{T}} \cdot \mathbf{U}=\left(\mathbf{X}^{\mathbf{T}} \cdot \mathbf{P}-\frac{\partial S}{\partial \mathbf{X}} \cdot \mathbf{B}\right)^{T}=\mathbf{P}^{\mathbf{T}} \cdot \mathbf{X}-\mathbf{B}^{\mathbf{T}} \cdot\left(\frac{\partial S}{\partial \mathbf{X}}\right)^{T} \\
\mathbf{U}=\left(\mathbf{R}^{\mathbf{T}}\right)^{-1} \cdot\left(\mathbf{P}^{\mathbf{T}} \cdot \mathbf{X}-\mathbf{B}^{\mathbf{T}} \cdot\left(\frac{\partial S}{\partial \mathbf{X}}\right)^{T}\right)
\end{gathered}
$$

The resulting expressions and substitute into the Bellman equation, while we seek its solution in the form:

$$
S(\mathbf{X}(t))=\mathbf{X}^{\mathbf{T}}(t) \cdot \mathbf{M}(t) \cdot \mathbf{X}(t),
$$

where: $\mathbf{M}(t)$ is an unknown symmetric nonstationary matrix of dimension $[n \times n] ; n$ - the order of the system under study.

The expressions needed for further transformations are written in the form:

$$
\begin{gathered}
\frac{\partial S}{\partial \mathbf{X}(t)}=2 \cdot \mathbf{X}^{\mathbf{T}}(t) \cdot \mathbf{M}(t) \\
\frac{\partial S}{\partial t}=\mathbf{X}^{\mathbf{T}}(t) \cdot \dot{\mathbf{M}}(t) \cdot \mathbf{X}(t)
\end{gathered}
$$




$$
\begin{gathered}
\mathbf{U}^{\mathbf{T}}=\mathbf{X}^{\mathbf{T}} \cdot \mathbf{P}-\mathbf{X}^{\mathbf{T}} \cdot \mathbf{M} \cdot \mathbf{B}=\mathbf{X}^{\mathbf{T}} \cdot(\mathbf{P}-\mathbf{M} \cdot \mathbf{B}) \\
\mathbf{U}=(\mathbf{P}-\mathbf{M} \cdot \mathbf{B})^{\mathbf{T}} \cdot \mathbf{X} .
\end{gathered}
$$

After carrying out the appropriate substitutions, we obtain the Bellman equation in the form:

$$
\begin{array}{r}
\mathbf{X}^{\mathrm{T}} \dot{\mathbf{M}} \mathbf{X}=\mathbf{X}^{\mathrm{T}} \mathbf{Q} \mathbf{X}+\mathbf{X}^{\mathbf{T}} \cdot(\mathbf{P}-\mathbf{M B})(\mathbf{P}-\mathbf{M B})^{\mathrm{T}} \mathbf{X}- \\
-2 \mathbf{X}^{\mathrm{T}} \mathbf{P}(\mathbf{P}-\mathbf{M B})^{\mathrm{T}} \mathbf{X}+2 \mathbf{X}^{\mathrm{T}} \mathbf{M} \mathbf{A} \mathbf{X}+2 \mathbf{X}^{\mathrm{T}} \mathbf{M B}(\mathbf{P}-\mathbf{M B})^{\mathrm{T}}
\end{array}
$$

From the last equality it is obviously possible to obtain an equation for determining the matrix $\mathbf{M}$ :

$$
\begin{aligned}
\dot{\mathbf{M}}=\mathbf{Q}+2 \mathbf{M A} & +(\mathbf{P}-\mathbf{M B})(\mathbf{P}-\mathbf{M B})^{\mathbf{T}}-2 \mathbf{P}(\mathbf{P}-\mathbf{M B})^{\mathbf{T}}+ \\
& +2 \mathbf{M B}(\mathbf{P}-\mathbf{M B})^{\mathbf{T}}
\end{aligned}
$$

Conducting the transformations of the last expression, we have:

$$
\begin{gathered}
\dot{\mathbf{M}}=\mathbf{Q}+2 \mathbf{M A}+(-2 \mathbf{P}+2 \mathbf{M B}+(\mathbf{P}-\mathbf{M B})) \cdot(\mathbf{P}-\mathbf{M B})^{\mathbf{T}} \\
\dot{\mathbf{M}}=\mathbf{Q}+2 \mathbf{M A}+(\mathbf{M B}-\mathbf{P}) \cdot(\mathbf{P}-\mathbf{M B})^{\mathbf{T}}
\end{gathered}
$$

Thus, we have obtained a nonlinear quadratic equation with respect to the matrix $\mathbf{M}(t)$.

Since the solution is sought in the quadratic form:

$$
S(\mathbf{X}(t))=\mathbf{X}^{\mathbf{T}}(t) \mathbf{M}(t) \mathbf{X}(t),
$$

the solution of this equation, for $t=\tau$, must satisfy the condition $\mathbf{M}(\tau)=\mathbf{Q}$.

The object under study is stationary, which means that the matrix $\mathbf{M}$ is constant. Then the equation under study has the form:

$$
\mathbf{Q}+2 \mathbf{M A}-(\mathbf{P}-\mathbf{M B})(\mathbf{P}-\mathbf{M B})^{\mathbf{T}}=0 .
$$

We perform fairly simple transformations:

$$
\begin{gathered}
(\mathbf{P}-\mathbf{M B})(\mathbf{P}-\mathbf{M B})^{\mathrm{T}}=\mathbf{P P}^{\mathrm{T}}-\mathbf{M B P}^{\mathrm{T}}- \\
-\mathbf{P B}^{\mathrm{T}} \mathbf{M}^{\mathrm{T}}+\mathbf{M B B}^{\mathrm{T}} \mathbf{M}^{\mathrm{T}} ; \\
(\mathbf{P}-\mathbf{M B})(\mathbf{P}-\mathbf{M B})^{\mathrm{T}}=\mathbf{P P}^{\mathrm{T}}-\mathbf{M B P}^{\mathrm{T}}- \\
-\mathbf{M}\left(\mathbf{P B}^{\mathrm{T}}\right)^{\mathrm{T}}+\mathbf{M B B}^{\mathrm{T}} \mathbf{M}^{\mathrm{T}} ; \\
(\mathbf{P}-\mathbf{M B})(\mathbf{P}-\mathbf{M B})^{\mathrm{T}}=\mathbf{P P}^{\mathrm{T}}-2 \mathrm{MBP} \mathbf{P}^{\mathrm{T}}+ \\
+\mathbf{M B B}^{\mathrm{T}} \mathbf{M}^{\mathrm{T}} .
\end{gathered}
$$

The final equation can be written as:

$$
\mathbf{Q}+\mathbf{P P}^{\mathbf{T}}-2 \mathbf{M}\left(\mathbf{A}-\mathbf{B} \mathbf{P}^{\mathbf{T}}\right)+\mathbf{M B B} \mathbf{B}^{\mathbf{T}} \mathbf{M}^{\mathbf{T}}=0
$$

\section{Conclusion}

Thus, the matrix equation is obtained, the solution of which will allow to determine the matrix and will make it possible to synthesize the control law according to the specified criterion.

\section{References}

1. F. Vasilyev, Optimization methods (Moscow, Factorial Press, 2002)

2. Fond, S., Optimal feedback control policies for stochastic-parameter systems, Journal of Optimization Theory and Applications, 30(3), 501512 (1980)

3. A. Alexandrov Optimal and adaptive systems, (Moscow, Higher School, 1990)

4. N. Polovinchuk, V. Trofimenko, N. Rudenko, S. Ivanov Optimal control of a stochastic dynamical system under terminal constraints, Dual technologies, 4, 40-43 (2013)

5. E.M. Galeev, Zelikin M.I., Konyagin, S.V. Osmolovsky, Optimum control (Moscow, MCNMO, 2008)

6. V. Komissarchik Methods of optimization and optimal control (Tver state technical University, 2002)

7. D. White, Optimality and Efficiency (Wiley, New York, 1982)

8. V. Surkov, Analytical design of optimal controllers by the criteria of accuracy, speed, energy saving (Tula State University, 2005)

9. D. Sue, A. Meyer, Modern theory of automatic control and its application (Moscow, Mechanical engineering, 1972)

10. V. Miroshnichenko, Research of dynamic characteristics of metal-cutting machine components at the design stage (Rostov-on-Don, DSTU Publishing Center, 2006)

11. V. Miroshnichenko, Problems of Synthesis of Modal Regulators of Machine Drives, (Rostov-on-Don, DSTU Publishing Center, 2009)

12. A. Balakrishnan Applied functional analysis (Moscow, Nauka, 1980)

13. F. Gontmakher, Matrix Theory (Moscow, Nauka, 1988)

14. R. Bellman, Introduction to the theory of matrices (Moscow, Mir, 1969)

15. A. Egorov On the matrix Riccati equations, Differ. equations, 1, (1995)

16. R. Bellman, On the theory of dynamic programming, Proceedings of the National Academy of Sciences, 38, 716-719 (1952)

17. R. Bellman, The theory of dynamic programming, Bulletin of the American Mathematical Society, 60, 503-515 (1954)

18. Y. Maksimov, E. Filipovskaya Algorithms for solving nonlinear programming problems (Moscow: MEPhI, 1982)

19. M. Sniedovich, Dynamic Programming (Marcel Dekker, New York, 1992)

20. M. Puterman, Dynamic Programming and its Application (Academic Press, Ney York, 1978)

21. T. Abdul-Razaq, C. Potts Dynamic programming state-space relaxation for single-machine scheduling, Journal of the Operational Research Society, 39(2), 141-152 (1988) 